\title{
Chemodynamical Modelling of Galaxy Formation and Evolution
}

\author{
Peter Berczik (berczik@mao.kiev.ua) \\ ${ }^{1}$ Main Astronomical Observatory of Ukrainian National Academy of Sciences, \\ Zabolotnoho Str. 27, Kiev, Ukraine \\ ${ }^{2}$ Astronomisches Rechen-Institut, Monchhofstrasse 12-14, Heidelberg, Germany \\ Gerhard Hensler and Christian Theis \\ Institut für Theoretische Physik und Astrophysik, University of Kiel, \\ Olshausenstr. 40, Kiel, Germany \\ Rainer Spurzem \\ Astronomisches Rechen-Institut, Monchhofstrasse 12-14, Heidelberg, Germany
}

November 28, 2001

\begin{abstract}
.
We present our recently developed 3-dimensional chemodynamical code for galaxy evolution. This code follows the evolution of different galactic components like stars, dark matter and different components of the interstellar medium (ISM), i.e. a diffuse gaseous phase and the molecular clouds. Stars and dark matter are treated as collisionless N-body systems. The ISM is numerically described by a smoothed particle hydrodynamics (SPH) approach for the diffuse gas and a sticky particle scheme for the molecular clouds. Additionally, the galactic components are coupled by several phase transitions like star formation, stellar death or condensation and evaporation processes within the ISM.

As an example we show the dynamical and chemical evolution of a star forming dwarf galaxy with a total baryonic mass of $2 \cdot 10^{9} M_{\odot}$. After a moderate collapse phase the stars and the molecular clouds follow an exponential radial distribution, whereas the diffuse gas shows a central depression as a result of stellar feedback. The metallicities of the galactic components behave quite differently with respect to their temporal evolution as well as their radial distribution. Especially, the ISM is at no stage well mixed.
\end{abstract}

Keywords: SPH, chemodynamics, dwarf galaxy evolution

\section{The 3d chemodynamical code and first results}

Since several years smoothed particle hydrodynamics (SPH) calculations have been applied successfully to study the formation and evolution of galaxies. Its Lagrangian nature as well as its easy implementation together with standard N-body codes allows for a simultaneous description of complex dark matter-gas-stellar systems (e.g. Navarro \& White, 1993; Mihos \& Hernquist, 1996). Here we present simulations based on our $3 \mathrm{~d}$ chemodynamical code. This code includes many complex effects such as a multi-phase ISM, cloud-cloud collisions, a drag force between different ISM components, condensation and evaporation

(C) 2018 Kluwer Academic Publishers. Printed in the Netherlands. 

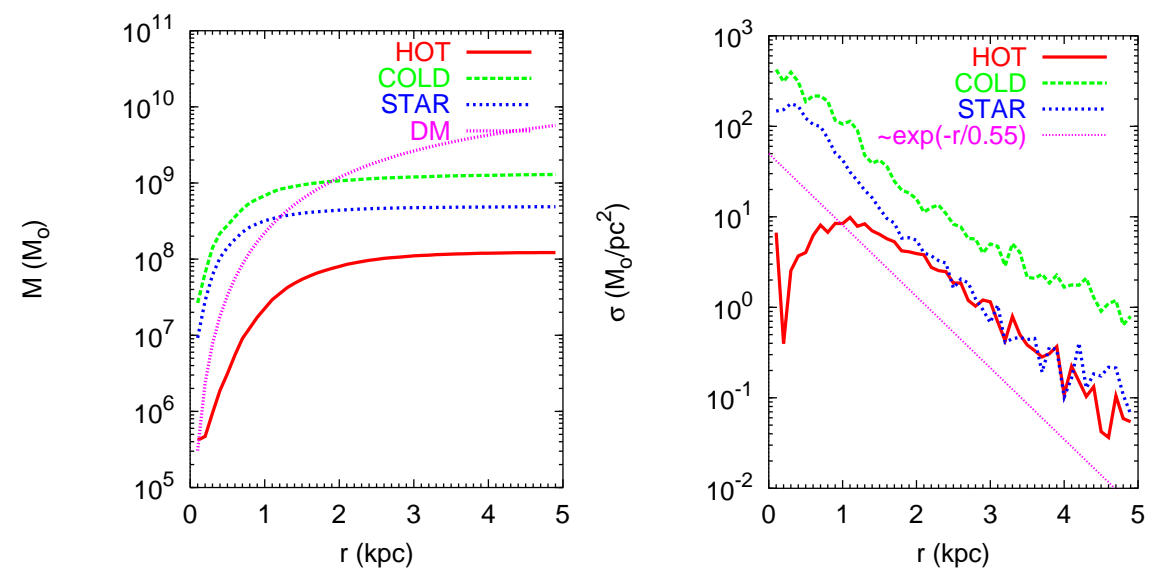

Figure 1. The radial distribution of the cumulative mass (left) and the surface density (right) for the different components in the central region of the model galaxy after 1 Gyr.

of clouds (CE), star formation (SF) and a stellar feedback (FB). This code is a further development of our single phase galactic evolutionary program (Berczik, 1999; Berczik, 2000) including now different gaseous phases.

In our new (multi-phase gas) code we use a two component gas description of the ISM (cold "clouds" and "smooth" hot SPH phase) (Theis et al., 1992; Samland et al., 1997). The basic idea is to add a cold cloudy component to the smooth and hot gas $\left(10^{4}-10^{7} \mathrm{~K}\right)$ described by SPH. The cold clumps are modeled as N-body particles with some "viscosity" (Theis \& Hensler, 1993) (cloud-cloud collisions and drag force between clouds and hot gas component). The cloudy component interacts with the surrounding hot gas also via condensation and evaporation processes (Cowie et al., 1981; Köppen et al., 1998). In the code we introduce also star formation. The "stellar" particles are treated as a dynamically separate N-body component. The cloud component forms stars. These stars return chemically enriched gas material and energy to both gaseous phases.

As a test of our new code, we calculate the evolution of an isolated star forming dwarf galaxy. The initial total gas content of our dwarf galaxy is $2 \cdot 10^{9} \mathrm{M}_{\odot}(80 \%$ "COLD" $+20 \%$ "HOT") which is placed inside a fixed dark matter halo with parameters $\mathrm{r}_{0}=2 \mathrm{kpc}$ and $\rho_{0}=0.075$ $\mathrm{M}_{\odot} / \mathrm{pc}^{3}$, using density profile of Burkert, 1995. With these parameters the dark matter mass inside the initial distribution of gas $(20 \mathrm{kpc})$ is $\approx 2 \cdot 10^{10} \mathrm{M}_{\odot}$. For the initial gas distribution we use a PlummerKuzmin disk with parameters $\mathrm{a}=0.1 \mathrm{kpc}$ and $\mathrm{b}=2 \mathrm{kpc}$ (Miyamoto \& Nagai, 1975). The gas initially rotates in centrifugal equilibrium around 

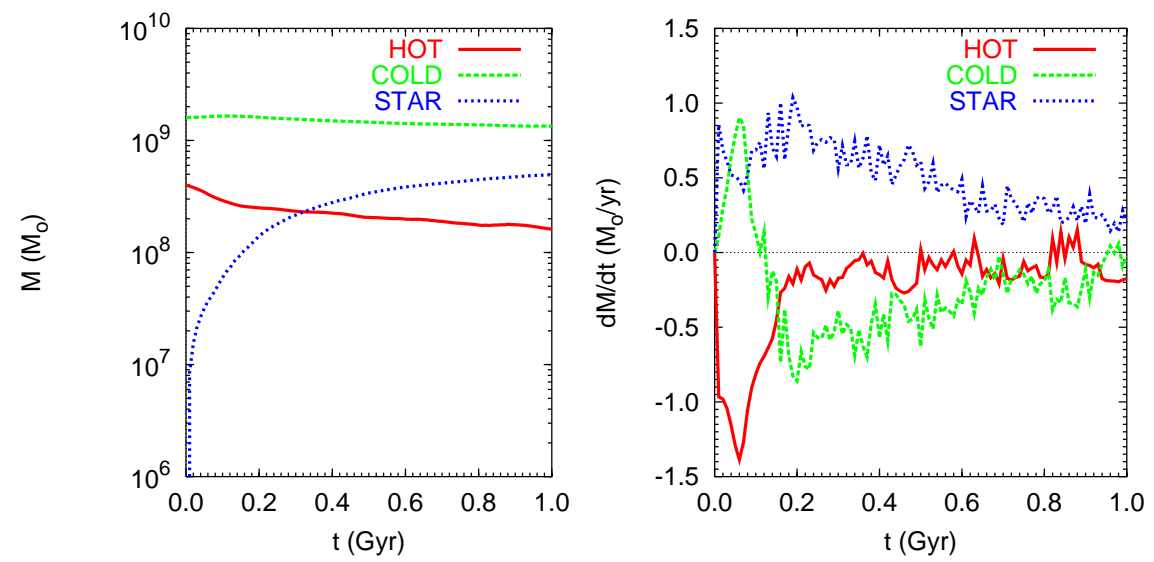

Figure 2. The temporal evolution of the mass (left) and mass exchange rate (right) for the different components of the model galaxy.

the z-axis. We choose the dwarf galaxy as an appropriate object for our code, because in this case even with a relatively "small" number of cold "clouds" $\left(\sim 10^{4}\right)$ we achieve the required physical resolution for a realistic description of individual molecular clouds $\left(\sim 10^{5} \mathrm{M}_{\odot}\right)$ as a separate "COLD" particle. In the simulation we use $\mathrm{N}_{\text {hot }}=10^{4}$ $\mathrm{SPH}$ and $\mathrm{N}_{\text {cold }}=10^{4}$ "COLD" particles. After 1 Gyr more then $10^{4}$ additional stellar particles are created.

In Fig. 1 we present the mass and surface density distribution of the different components in the central region of the model after 1 Gyr of evolution. In the region up to $\approx 2 \mathrm{kpc}$ the baryonic matter dominates over the DM. The surface density of the stars can be well approximated by an exponential disk with a scale length of $0.55 \mathrm{kpc}$. In the distribution of hot gas, we see a central "hole" ( $\approx 1 \mathrm{kpc})$, as a result of gas blow-out from the center mainly due to SNII explosions.

In Fig. 2 we present the evolution of the mass and the mass exchange rate of the different components. The SFR (i.e. $\mathrm{dM}_{\mathrm{STAR}} / \mathrm{dt}$ ) peaks to a value of $1 \mathrm{M}_{\odot} \mathrm{yr}^{-1}$ after 200 Myrs. Afterwards it drops down to 0.2 $\mathrm{M}_{\odot} \mathrm{yr}^{-1}$ within several hundred Myrs. Another interesting feature is the behaviour of the hot gas phase mass exchange. After the initial violent phase of condensation an equilibrium is established which gives a hot gas fraction of about $10 \%$ of the total gas mass.

The metal content of the diffuse gas and the clouds differs significantly over the whole integration time (Fig. 3). Due to SNII and SNIa events the metallicity of the hot phase exceeds that of the clouds by almost one order of magnitude. The clouds mainly get their metals by condensation of the hot phase. The central metallicity plateau (up to $1 \mathrm{kpc}$ ) of the cold component is explained by the fact, that condensation 
$N$

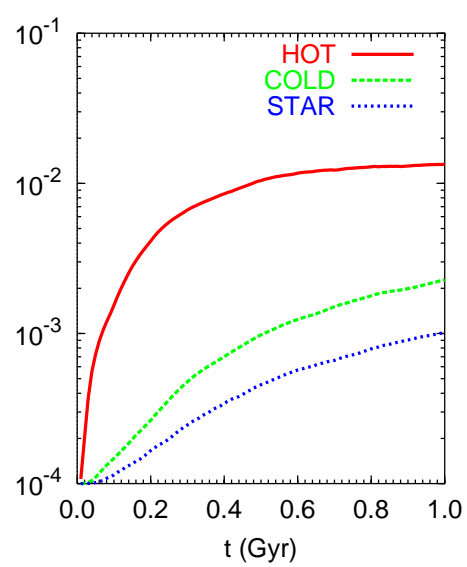

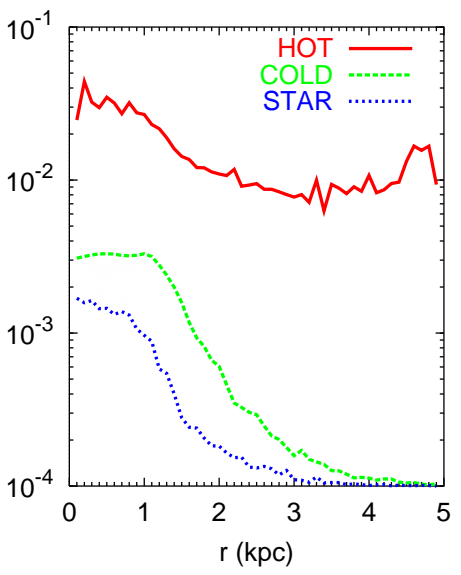

Figure 3. Temporal evolution of the metallicities (left) and their radial distribution after 1 Gyr (right). Individual metallicities of newly born stars are marked by dots.

is not very efficient in that region, e.g. because of the central "hole" of the very hot diffuse ISM and a lack of metal enriched material there. Moreover, the conditions in the center lead mainly to evaporation of clouds which also prevents the mixing with the metal enriched hot gas.

\section{Acknowledgements}

The work was supported by the German Science Foundation (DFG) with the grants 436 UKR 18/2/99, 436 UKR 17/11/99 and the SFB 439 (University of Heidelberg). P.B. is grateful for the hospitality of the Astronomisches Rechen-Institut (Heidelberg), where the main part of this work has been done. The numerical models have been computed with the GRAPE-5 system at the Astronomical Data Analysis Center of the National Astronomical Observatory, Japan.

\section{References}

Berczik P., 1999, A\&A, 348, 371

Berczik P., 2000, Ap\&SS, 271, 103

Burkert A., 1995, ApJ, 447, L25

Cowie L.L., McKee C.F. \& Ostriker J.P., 1981, ApJ, 247, 908

Köppen J., Theis Ch. \& Hensler G., 1998, A\&A, 331, 524

Mihos J.C. \& Hernquist L., 1996, ApJ, 464, 641

Miyamoto M. \& Nagai R., 1975, PASJ, 27, 533

Navarro J.F. \& White S.D.M., 1993, MNRAS, 265, 271

Samland M., Hensler G. \& Theis Ch., 1997, ApJ, 476, 544

Theis Ch., Burkert A. \& Hensler G., 1992, A\&A, 265, 465

Theis Ch. \& Hensler G., 1993, A\&A, 280, 85 